\title{
Le Premier Congrès d'Interasma à Lübeck et Bad \\ Lippspringe
}

\section{2 au 15 juillet 1956}

La premiere reunion scientifique, organisée par ГAssociation Internationale d'Asthmologie (Interasma), a eu lieu les 12 et 13 juillet au Krankenhaus Süd de Lübeck, sous la présidence du Professeur Karl Hansen.

Cette reunion avait surtout été organisée pour discuter des moyens propres à aider le développement futur de l'Association, mais la partie scientifique, organisée de main de maître par le Prof. Hansen et ses collaborateurs, les Drs. Schliack, Lubbers, Sudeck, Evers, Stresemann, Ernst et Trabulsí, fut presque entièrement consacrée à la demonstration des divers moyens du diagnostique allergique et notamment, les reactions de Prausnitz-Küstner obtenues soit après injection soit après ingestion ou inhalation de Pallergène par le sujet dont une region du derme avait été préalablement sensibilisé.

Sous $\Gamma$ égide de Schwester Charlotte Kohncke, la compétente directrice du Labora-toire des Tests, les congressistes furent instruits de toutes les méthodes originales qui y ont été élaborées pour l'extraction, la purification et la conservation des extraits d'al-lergènes.

Les deux jours suivants, les congressistes furent les hôtes du Dr. W. Gronemeyer, directeur de Plnstitut des Recherches sur l'Asthme et $\Gamma$ Allergie à Bad-Lippspringe (Westphalie). Après visite des installations thermales de la station où plusieurs centaines d'asthmatiques (curistes privés ou patients hospitalises grace à la Sécurité sociale) sont soignés durant toute 1'année par les thérapeutiques anti-asthmatiques les plus modernes, les participants suivirent les demonstrations, effectuées par le Dr. E. Fuchs, de recherche de la sensibilité des muqueuses bronchiques aux pneumallergènes par la méthode de la pneumométrie d'après Wyss et Hadorn. (Bibliographie: voir Fuchs, E., und W. Gronemeyer: Zur Analyse nächtlicher Anfallsauslösung beim Asthma bronchiale. Munch, med. Wschr. No. 15, 1956.)

Le Dr. Nikkei (Bad Lippspringe) résuma ensuite les divers moyens thérapeutiques utilises dans la station. Outre les traitements spécifiques, qui impliquent la nécessité d'un diagnostic étiologique d'une grande precision, le médecin pourra prescrire, suivant les nécessités, les aerosols broncho-dilatateurs ou detergents, chauds ou froids, avec application d'ondes courtes en cas d'infection chronique. II est fait usage de chambres d'inhalations dans lesquelles le malade pourra respirer soit des pulverisations d'eau salée hypertonique à $2 \%$ qui agissent comme sécrétolytique, soit des pulverisations d'acides organiques qui améliorent surtout les emphysémateux. Comrne l'a montré le

Proceedings - Sociétés - Gesellschaftsberichte

Professeur von Hayek chez Tanimal, les acides organiques, provoquant la retraction des cellules de $\Gamma$ epithelium alvéolaire, facilitent les échanges entre le sang et $\Gamma$ air des alveoles.

La cure «climatique» est effectuée dans des caissons dont Гatmosphère, dépourvue d'allergènes, la temperature et la pression peuvent être modifiées suivant les indications thérapeutiques.

Citons enfin, la gymnastique respiratoire, active ou bien passive par courant à basse frequence excitant la musculature abdominale synchroniquement avec l'ex-piration. 
Si le massage par kinésistes s'est révélé un moyen précieux pour «délier» les causes du bronchospasme, le massage mécanique rythmique par ventouses à depression variable lui est supérieur. II accélère la circulation sanguine de la peau et realise ainsi une manière de lavage du tissu conjonctif.

Ajoutons, parmi les autres moyens physiothérapiques utilises couramment à Bad Lippspringe, les ondes courtes, les rayons U.V., les massages sous eau, les douches de Kneipp, les irrigations coloniques et les bains médicamenteux.

Outre les participants déjà mentionnés, ce premier Congrès, reserve aux membres fondateurs d'Interasma, avait groupé MM. Sanchez-Cuenca (Madrid), Damas-Mora (Lisbonne), Gau (Grasse), Duchaine (Bruxelles), Quarles van Ufford (Utrecht), Mrs. Walker (Oxford), Sugihara (Tokyo), Centanni (Milan), Businco (Rome), Kallós (Helsingborg), van der Bijl (Amsterdam), Findeisen (Dresden), Michel (Marbourg), Werner (Hambourg).

Malheureusement, le Professeur Sangiorgi, Гinfatigable secrétaire-général d'Interasma, retenu à Milan par une grave maladie, ne put se rendre à Lübeck où son absence fut unanimement déplorée au moment où son «enfant chéri» était tenu sur les fonds baptismaux.

Deux banquets réunirent les congressistes à Travemünde d'abord, à Bad Lippspringe ensuite et permirent aux délégués nationaux d'exprimer leur reconnaissance au Professeur Hansen et au Dr. Gronemeyer pour la parfaite réussite du Congrès.

Enfin, Mesdames Hansen et Gronemeyer, firent avec infiniment de grace, les hon-neurs de leur ville et leurs hôtes purent goûter pendant quelques instants, trop courts à leur gré, le charme prenant de la vieille et fière cite hanséatique.

J. Duchaine, Bruxelles. 